\title{
Stable Flatbands, Topology, and Superconductivity of Magic Honeycomb Networks
}

\author{
Jongjun M. Lee $\odot,{ }^{1}$ Chenhua Geng, ${ }^{2}$ Jae Whan Park, ${ }^{3}$ Masaki Oshikawa, ${ }^{2}$ Sung-Sik Lee, ${ }^{4,5}$ \\ Han Woong Yeom, ${ }^{3,1}$ and Gil Young Cho, ${ }^{1, *}$ \\ ${ }^{1}$ Department of Physics, Pohang University of Science and Technology (POSTECH), Pohang 37673, Republic of Korea \\ ${ }^{2}$ Institute for Solid State Physics, The University of Tokyo, Kashiwa, Chiba 277-8581, Japan \\ ${ }^{3}$ Center for Artificial Low Dimensional Electronic Systems, Institute for Basic Science (IBS), Pohang 37673, Korea \\ ${ }^{4}$ Department of Physics \& Astronomy, McMaster University, 1280 Main St. W., Hamilton Ontario L85 4M1, Canada \\ ${ }^{5}$ Perimeter Institute for Theoretical Physics, 31 Caroline ST. N., Waterloo Ontario N2L 2Y5, Canada
}

(Received 18 August 2019; revised manuscript received 8 November 2019; accepted 6 March 2020; published 1 April 2020)

\begin{abstract}
We propose a new principle to realize flatbands which are robust in real materials, based on a network superstructure of one-dimensional segments. This mechanism is naturally realized in the nearly commensurate charge-density wave of $1 \mathrm{~T}-\mathrm{TaS}_{2}$ with the honeycomb network of conducting domain walls, and the resulting flatband can naturally explain the enhanced superconductivity. We also show that corner states, which are a hallmark of the higher-order topological insulators, appear in the network superstructure.
\end{abstract}

DOI: 10.1103/PhysRevLett.124.137002

Band theory of electrons has gone through a renaissance in recent years, especially concerning its topological nature $[1,2]$. Band structures are also an important starting point to understand strongly correlated systems. In particular, when the Fermi level lies in a flatband, the correlation effects become dominant, leading to many interesting physics including superconductivity and magnetism. A few theoretical principles to realize flatbands are known, most notably the chiral (sublattice) symmetry and an imbalance between the number of sublattice sites $[3,4]$. However, once further nearest-neighbor hoppings, which generally exist in real materials, are included, the chiral symmetry is lost and the flatbands in the simplified model acquire a substantial curvature. This is a major reason why it has been difficult to realize flatbands in real materials and observe resulting strong correlation effects experimentally, with few exceptions [5-7].

In this Letter, we propose a novel general principle to realize flatbands based on a network of one-dimensional segments. The resulting flatbands are protected by the combination of crystal and time-reversal symmetries, and are robust even in the presence of the further neighbor hoppings, as long as the effective hopping range is shorter than the segment length. We argue that this mechanism is naturally realized in the nearly commensurate chargedensity wave (NC-CDW) phase of $1 \mathrm{~T}-\mathrm{TaS}_{2}$, in which the domain walls play the role of the one-dimensional metallic segments [8]. The robust flatbands ensured by the new principle give a natural explanation of the observed superconductivity, which is strongly reminiscent of the moiré physics [5-7] of twisted bilayer graphene. Moreover, the network superstructure can also lead to corner states, i.e., a higher-order topological insulator.
Experimentally, 1T- $\mathrm{TaS}_{2}$ has a rich phase diagram of CDW orders [9] and superconductivity (SC) [10-12], which are accessible by tuning temperature, pressure, and doping. There are three distinct regimes: commensurate $\mathrm{CDW}$ (C-CDW), NC-CDW, and incommensurate CDW. The C-CDW phase has a long-ranged charge ordering and appears at the lowest temperature with the ambient pressure. This state is the correlation-driven Mott insulator [13-15]. When the C-CDW ordering is slightly suppressed by pressure or doping, then the domain walls appear in between the locally charge-ordered domains, i.e., it enters into the NC-CDW state. If the pressure or doping increase further, the SC emerges from this NC-CDW state. Note that a similar phase diagram is obtained in another CDW material, namely, 1T-TiSe 2 [16]. This suggests that the NCCDW $[10-12,16,17]$ is somehow essential for realizing SC in these CDW materials though how this actually happens has been unclear. We will point out that a honeycomb network of metallic domain walls in the NC-CDW phase $[8,18,19]$ hosts a series of robust flatbands ensured by the new principle, giving a natural explanation for the observed SC.

1. Model and flatbands: A recent STM study [8] combined with DFT calculation provided an unprecedented detail of the electronic structure of the network, where the metallic nature of the domain walls and trijunctions is clearly exposed. Similar structure emerges in, e.g., helium mixture absorbed to graphite [20]. Here we consider a minimal tight-binding model, which consists of the lowenergy modes inside the domain walls; see Fig. 1(a).

$$
H_{0}=-t_{0} \sum_{\left\langle\boldsymbol{r}, \boldsymbol{r}^{\prime}\right\rangle} c_{\boldsymbol{r}, \sigma}^{\dagger} c_{\boldsymbol{r}^{\prime}, \sigma}-t_{J} \sum_{\left\{\boldsymbol{r}, \boldsymbol{r}^{\prime}\right\} \in J} c_{\boldsymbol{r}, \sigma}^{\dagger} c_{\boldsymbol{r}^{\prime}, \sigma}+\text { H.c., }
$$


(a)

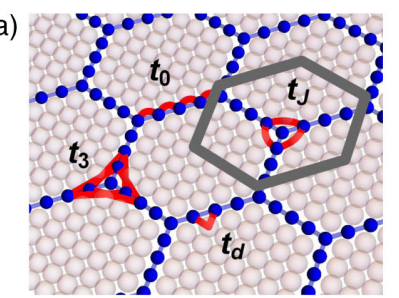

$(c)_{4}$

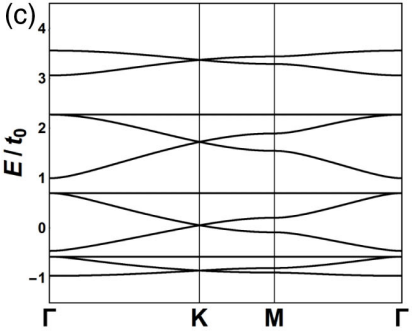

(b)

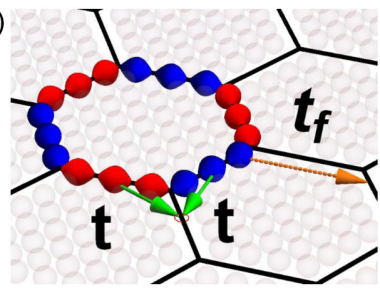

(d)

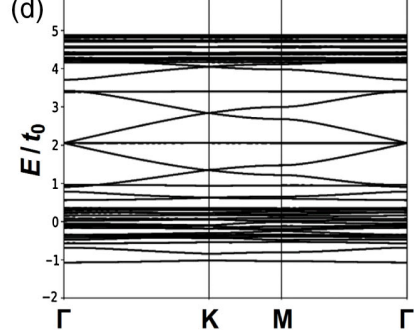

FIG. 1. (a) Network of 1D metals. In Eq. (1), only the sites along the straight blue lines are included. The other sites represent the insulating domain sites. The gray line represents the unit cell. (b) The states inside the flatbands. Blue and red colored regions have the opposite signs and a destructive interference appears for any hopping shorter than the link length (e.g., $t$ ). (c) Band structure of Eq. (1). (d) Band structure including the domain sites.

where the second sum over $\left\{\boldsymbol{r}, \boldsymbol{r}^{\prime}\right\} \in J$ runs over the sites around the junctions $J$ and $t_{J} \leq t_{0}$. Here $\sigma$ represents the spin and the sum over it is assumed. The spin rotational symmetry is also assumed for simplicity. The model also has time-reversal symmetry $\mathcal{T}$, and crystalline $D_{6}$ symmetry, which are the symmetries observed in the STM experiment of $1 \mathrm{~T}-\mathrm{TaS}_{2}$ [8]. Diagonalizing Eq. (1), we find a cascade of flatbands, Dirac and quadratic band crossings; see Fig. 1(c). On top of this, a three-component spin-1 Dirac fermion [21] can appear when $t_{J} / t_{0} \rightarrow 0$. The number of the flatbands is proportional to the number of the sites between the junctions. The topological band crossings are protected by symmetries. On the other hand, the flat dispersion cannot be generally protected because it requires infinitely many parameters to be tuned. Hence, they are generically fragile, e.g., against the second neighbor hoppings [22]. Despite of the fragile nature, flatbands are an ideal stage to realize correlation-dominated physics, such as ferromagnetism, and thus have been studied vigorously [33]. A well-known mechanism which gives rise to flatbands is an imbalance between sublattice sites in bipartite lattices. This was applied [3] to hyperhoneycomb systems which have some similarities with the systems we study in this Letter. However, such flatbands can be generically removed by inclusion of short-ranged further neighbor hoppings, which exist in real materials.

Remarkably, our flatbands from the network defy this standard phenomenology and are stable against $D_{6^{-}}$ symmetric local perturbations. For example, addition of the third-neighbor hoppings $t_{3}$ near the nodes do not disperse the flatbands. We can even include the insulating

electronic states from the domain area (described by $H_{\text {dom }}$ ); see Fig. 1(a). The full Hamiltonian is now $H=H_{0}+$ $H_{\text {dom }}+H_{\text {coup }}$, with

$$
H_{\text {coup }}=-t_{d} \sum_{\left\langle\boldsymbol{r}, \boldsymbol{r}^{\prime}\right\rangle} c_{\boldsymbol{r}, \sigma}^{\dagger} d_{\boldsymbol{r}^{\prime}, \sigma}+\text { H.c., }
$$

where $c_{r, \sigma}\left(d_{r, \sigma}\right)$ electrons belong to the network (to the domains). Here $H_{\text {dom }}$ is described by the band insulator which has the two energy-split states per site and the different sites are connected by the small hopping $t_{d}$ [Fig. 1(a)]. From the perturbative reasoning, we expect that this model includes all the possible symmetric local perturbations. Notably, the flatbands and overall band shapes remain almost intact inside the gap [Fig. 1(d)]. This result implies that in NC-CDW $1 \mathrm{~T}-\mathrm{TaS}_{2}$, even if the bulk bands from the domain region are included, flatbands inside the gap are almost intact. Finally, the flatbands survive [22] against the Rashba spin-orbit coupling. Such stability is absent for other networks [22]. In passing, we note that this is consistent with our previous phenomenological approach; see Ref. [22].

To explain the unusual stability, we look into the structure of the wave functions inside the flatbands. We find that those wave functions vanish at the junctions [22]. Hence, when the low-energy modes are entirely from the network and only the nearest neighbor hoppings are included, i.e., $t_{J}=0$ in Eq. (1), the wave function is a standing wave $\psi(l)$ [Fig. 1(b)]. From such standing waves, we can construct a set of localized states [34] which consist of the flatbands: we consider a linear combination around the honeycomb plaquette with a sign oscillation, i.e., $\Psi \sim \sum_{a}(-1)^{a} \psi(l)$ for $a \in\{1,2, \ldots 6\}$ labeling the six links around the plaquette; see Fig. 1(b). Then we see that this state cannot disperse into the neighboring plaquettes because of the destructive interference. Such destructive interference persists as far as the hopping distance is shorter than the length of the wire. Finally, the intrawire further neighbor terms do not alter this conclusion because they affect only the energy and intrawire structure of the standing waves [22]. This illustrates the importance of the symmetry and the locality on the stability of the flatbands. We can also understand that the cascade of the flatbands must appear because there are many standing wave solutions per wire [35].

We expect the flatbands to be removed either by longranged direct hopping across the domains or by breaking symmetries. Indeed, we can show [22] that the flatbands are lifted when the symmetries are broken, or when such longranged hoppings [e.g., $t_{f}$ in Fig. 1(b)] are included. This means that, for the NC-CDW state of $1 \mathrm{~T}^{-\mathrm{TaS}_{2}}$, we need a sizable hopping between the two sites apart by $\sim O(80) \AA$ [8] to remove the flatbands, which is not realistic. This explains why the dispersion of the "flatbands" are very small but still finite when the domain sites are included. When the time-reversal symmetry is broken, the band 
touchings are gapped out and it results in dispersive Chern bands [22], which is a natural platform for fractionalization [36].

For $1 \mathrm{~T}-\mathrm{TaS}_{2}$, we constructed a tight-binding model in the Supplemental Material [22], which fits reasonably well with DFT $+U$ calculation on the domain wall. The tightbinding parameters scale as $1 / d^{5}$ [23], where $d$ is the distance between the atomic sites. From this, we find that the cascade of the flatbands emerges [22]. Next, we comment on the effect [37-40] of the interlayer coupling. The interlayer interaction has been suggested to be important in $1 \mathrm{~T}-\mathrm{TaS}_{2}$. However, we remark that there are plenty of experimental data and theory $[8,15]$ suggesting that the main physics is essentially $2 \mathrm{D}$. For example, the resistivity along the $c$ axis is much larger, e.g., by 500 times [41], than the intralayer resistivity [41,42] and anisotropic 2D charge transfer is observed for the NC-CDW state [43]. Further, the SC $T_{c}$ is almost insensitive to the pressure [10] and not much affected under the dimensional reduction [11]. Based on these, we focus on the 2D physics here.

2. Superconducting states: Having established the stability of the flatbands, we now discuss the many-body physics when the Fermi level is near one of the flatbands. Such a system is unstable toward various particle-hole and particle-particle channels. However, guided by experiments, we mainly focus on superconductivity in this Letter.

First, we perform the simplest BCS mean-field theory with the phenomenological interaction

$$
H_{\mathrm{int}}=U \sum_{r} n_{r}^{2}+V \sum_{\left\langle\boldsymbol{r}, \boldsymbol{r}^{\prime}\right\rangle} n_{r} n_{r^{\prime}}
$$

Projecting to the BCS channel, we obtain [22]

$$
H_{\mathrm{int}} \rightarrow H_{\mathrm{BCS}}=\sum_{l \in 2 \mathbb{Z}} g_{l} \sum_{\boldsymbol{p}, \boldsymbol{k}} \hat{\triangle}_{l ; p}^{\dagger} \cdot \hat{\triangle}_{l ; k},
$$

where $g_{l}$ is the interaction strength along the pairing channel of the angular momentum $l$, which we compute numerically. $\hat{\triangle}_{l ; p}$ is the corresponding pairing order parameter. Note that, within the mean-field decomposition of Eq. (2), only the spin-singlet sector appears. Below we consider only the $s$-wave and $(d+i d)$-wave pairing channels, i.e., $g_{0}$ and $g_{2}=g_{-2}$ of Eq. (3). Higher angular momentum pairing channels $(|l|>2)$ will belong to the same representations of $s$ - or $(d \pm i d)$-wave pairing channels in the honeycomb symmetry. The magic of the flatbands appears when the gap equation is solved.

$$
\frac{1}{g_{l}} \sim \int_{\mathrm{BZ}} \frac{d^{2} k\left|F_{l}(\boldsymbol{k})\right|^{2}}{\sqrt{\left(\epsilon_{k}-\mu\right)^{2}+\left|F_{l}(\boldsymbol{k}) \triangle_{l}\right|^{2}}} \sim \frac{1}{\left|\triangle_{l}\right|},
$$

where $F_{l}(\boldsymbol{k})$ is the form factor [22], e.g., $F_{2}(\boldsymbol{k}) \rightarrow\left(k_{x}+\right.$ $\left.i k_{y}\right)^{2}$ for $|\boldsymbol{k}| \ll 1$ and $\epsilon_{\boldsymbol{k}}=\mu$. Hence, the gap is linearly enhanced, i.e., $\left|\triangle_{l}\right| \sim\left|g_{l}\right|$ (if $g_{l}<0$ ), instead of the standard
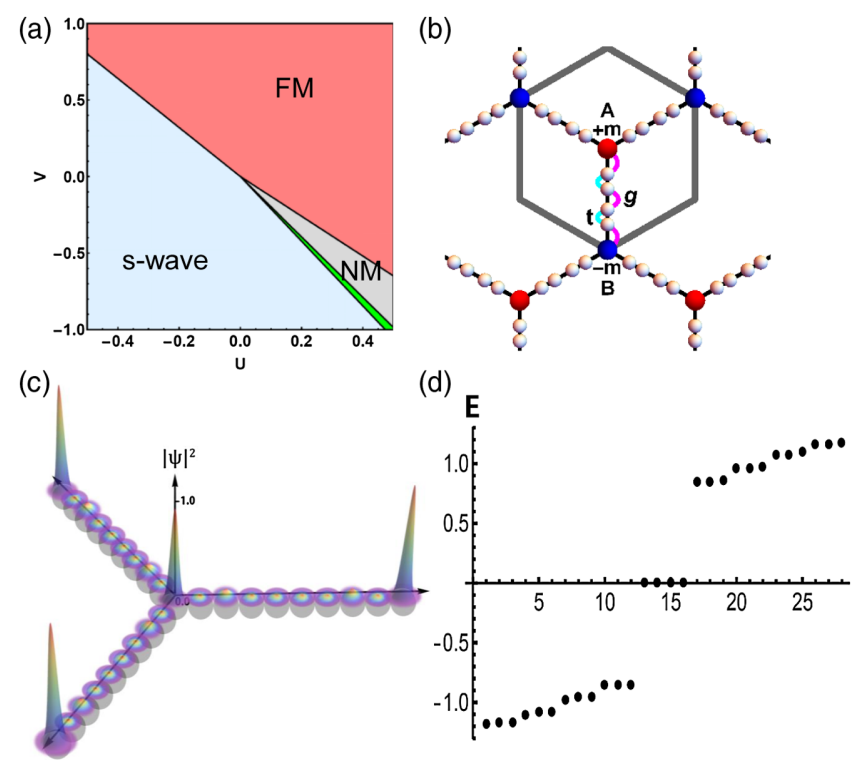

FIG. 2. (a) Phase diagram. " $s$-wave" represents an $s$-wave SC, and "FM" ("NM") represent ferromagnetic (normal metal) state. The green region is a $(d+i d)$-wave SC. (b) Pictorial representation of the model for the higher-order topology. Here $|t|<|g|$ supports the $0 \mathrm{D}$ state at the junctions and the corner at the boundary. The gray line represents the unit cell. (c) Density distribution of four in-gap modes of (d). (d) Energy eigenvalues for a single unit cell with open boundary condition for $t / g=0.1$.

exponential suppression $\sim \exp \left(-1 /\left|g_{l}\right| \nu_{l}\right)$. Because there is no other scale, the mean-field energy of the SC is linear in $g_{l}$ and so is the $T_{c}$ [22]. Hence, the honeycomb network strongly enhances the SC $T_{c}$. The phase diagram for one flatband is in Fig. 2(a). Thus we conclude that, within the mean-field theory, the $s$-wave SC is strongly enhanced when $U$ is attractive [44]. On the repulsive interaction side, the system exhibits ferromagnetism. While the repulsive $U$ opens up a small window for the $(d \pm i d)$-channel pairing, the dominance of the $s$-wave SC is a characteristic feature of the present flatband system [22]. In passing, the BdG fermion spectrum is also computed [22].

Although the mean-field theory ignores the fluctuation, it is a good starting point for revealing possible phases and phase diagram. In fact, the mean-field theory qualitatively agrees with a rigorous result on flatband ferromagnetism [45] with a repulsive $U$, and our findings in this Letter are consistent with recent numerical studies in other flatband systems $[44,46-48]$.

Let us comment on the interaction Eq. (2). The strong electron-phonon coupling in $1 \mathrm{~T}-\mathrm{TaS}_{2}$ is known as the driving force for the formation of the CDW states [49]. However, after the formation of the CDW clusters through the electron-phonon coupling, the soft phonons are naturally hardened [50]. In particular, the domain wall in the NC-CDW state is also structurally reconstructed by forming its own CDW clusters from electron-phonon coupling [8], which will reduce the coupling more. Hence, we expect 
that the electron-phonon coupling becomes inactive for the physics within the NC-CDW phase. Nevertheless, the phonon-electron interaction was essential in forming the parent CDW domains and the domain wall network, and hence its effect is already encoded the model Eq. (1). Next, it is well known that the coupling of electrons and (optical or gapped) phonons effectively plays the same role as the attractive $U$, which favors the $s$-wave SC [22]. This will effectively renormalize $U$ in Eq. (2) toward negative. Hence, explicitly including the effects of these phonons does not alter the dominance of the $s$-wave SC [22]. On the other hand, the long-range Coulomb interaction will be efficiently screened due to the large density of states of flatbands and it will be rendered into a short-ranged interaction, which can be effectively encoded by Eq. (2).

It is instructive to consider the strong-coupling limit [51], where the interaction is bigger than the hopping integrals. For this, we start from the decoupled strongly correlated wires, each of which is described by a Tomonaga-Luttinger liquid (TLL)

$$
H=\sum_{a, \sigma=c / s} \int d l \frac{v_{\sigma}}{2}\left[\frac{1}{K_{\sigma}}\left(\partial_{l} \theta_{a, \sigma}\right)^{2}+K_{\sigma}\left(\partial_{l} \phi_{a, \sigma}\right)^{2}\right],
$$

where the Luttinger parameters $\left\{K_{c}, K_{s}\right\}$ capture the correlation nonperturbatively [52]. This is the correlated version of the scattering problem [8], which faithfully reproduced the band structures.

The two-dimensional SC can be preempted by the spin gap $[52,53]$ in each wire. Once the spin gap forms, the low-energy physics of each wire is described by a singlecomponent TLL of $\theta_{a, c}$ describing the fluctuating SC pairs [52]. Since the SC pair is bosonic, the junction of three TLLs at each vertex of the honeycomb network corresponds to the bosonic $Y$ junction [54], rather than the fermionic one $[55,56]$ where the fermion statistics plays an important role. When each wire has sufficient attraction, i.e., $K_{c} \leq 1$ [52], then the interwire coupling between the SC fluctuations, namely, the Josephson coupling $J$, becomes relevant [52]. Only interested in the pattern of the phases, we note that the problem is symmetrically equivalent to the $X Y$ model on the kagome lattice, while leaving the full quasi-1D treatment [53] to the future study

$$
H_{\text {eff }}=J \sum_{\langle i, j\rangle}\left[e^{\sqrt{2 \pi} i\left(\theta_{i, c}-\theta_{j, c}\right)}+\text { H.c. }\right] .
$$

Depending on the sign of $J$, either the 2D $s$-wave or $(d \pm i d)$-wave SCs can emerge. When $J>0$, then the socalled $\sqrt{3} \times \sqrt{3}$ order appears [57], which translates as the $(d \pm i d)$-SC. If $J<0$, the conventional $s$-wave SC emerges. When the repulsive- $U$ dominates the junction region and the region becomes Mott insulating [58], $J>0$ can appear. From this strong-coupling limit, we can learn how the $2 k_{F}$-density wave of $1 \mathrm{D}$ wires competing with SC is suppressed. For the generic filling of each wire, the momentum $k_{F}$ will not be commensurate with the wire length $L$, i.e., $\phi_{L}=k_{F} L$ is not a rational number. This frustrates the phases of the density waves and thus their true two-dimensional order is strongly suppressed to develop. Because the density waves are the main competitors of the $\mathrm{SC}$ in one dimension, this gives a natural favor on the SC.

The domain wall states of $1 \mathrm{~T}-\mathrm{TaS}_{2}$ presumably experience small correlation effect and the junction regions are quite metallic from the STM data [8]. Furthermore, no magnetism is observed in experiments, i.e., interaction is dominantly attractive. Furthermore, the SC in $1 \mathrm{~T}-\mathrm{TaS}_{2}$ is experimentally observed for a broad range of the parameters $[10,11]$. When compared with Fig. 2(b), the SC is consistent with the $s$ wave. These suggest that the SC state of $1 \mathrm{~T}-\mathrm{TaS}_{2}$ is likely an $s$-wave SC.

3. Higher-order topology: When the filling per wire is commensurate, then the network can develop its own CDW order and become an insulator. We highlight here that the insulating network hosts an interesting possibility, namely, emergent corner states which are akin to those of the 2D higher-order topological insulators [24-26]. We illustrate this on the half-filled spinless fermion model, whose generalization to the spinful systems is straightforward. In such wires, the dimerization is expected, which manifests as the staggered nearest-neighbor hopping parameters; see Fig. 2(b). To clearly expose the corner states, we perform a finite-size calculation on a single unit cell of the network. In the spectrum Fig. 2(d), we see four in-gap modes, which are localized at the trijunction and the boundary Fig. 2(c). Those states are protected by crystal symmetries. We can also show that the above system similarly supports an in-gap mode at the corner of the edge. See Supplemental Material [22], where the junction of two gapped domain walls supports a corner mode protected by a reflection symmetry. Such OD states protected by crystalline symmetries are the hallmarks of higher-order topology.

The above higher-order topology of gapped domain wall networks may be relevant to various materials with similar structures such as $\mathrm{C}-\mathrm{CDW}$ 1T- $\mathrm{TaS}_{2}$ [27] and twin boundary networks of $\mathrm{MoSe}_{2}$ grown on $\mathrm{MoS}_{2}$ [59]. Spectacularly, it has been noted [23,27] that the insulating domain walls of C-CDW $1 \mathrm{~T}-\mathrm{TaS}_{2}$ have precisely the same structure as the dimerization, and they form junctions and networks. We believe that the confirmation of the higherorder topology in these systems will be an extremely interesting future problem, given that there is no concrete experimental demonstration of the $2 \mathrm{D}$ higher-order topology so far.

4. Conclusions: We considered the electronic structure of a conducting honeycomb network, where we uncovered the emergence of the cascade of the flatbands that are stable against various local perturbations. Compared to the previous studies $[3,33,34,60-63]$, our work reveals that the 
flatbands can emerge in much broader sets of the models beyond the models with chiral symmetry or only nearestneighbor hopping terms. We also demonstrated that the domain wall network is an ideal place to find diverse topological band structures: topological band crossings, and higher-order corner states. We find that the robust flatbands and network geometry can explain the coexistence of SC and CDW states in the network materials, which goes beyond the previous Landau-Ginzburg theory [17], which is blind to the emergent electronics from the network.

The signature of the network and its role in SC can be detected in various experiments in $1 \mathrm{~T}-\mathrm{TaS}_{2}$. First, the combination of our STM data, DFT calculation, [8] and the result of our current Letter already points strongly toward the existence of the flatbands. In particular, the scanning tunneling spectroscopy [8] showed that the band gap is filled, which again supports the emergence of cascade of band structures. In particular, one nearly flatband is observed right below the chemical potential in the currently available photoemission data [64-66] though further investigation will be desirable. Second, magnetotransport and oscillations can provide the information about the primary conducting and SC channels. They have been applied in the small twist-angle bilayer graphene [28,67] and textured superconducting states of $1 \mathrm{~T}-\mathrm{TiSe}_{2}[16,17]$. Also the flatbands show a few characteristic behaviors in thermodynamic quantities, which we summarize in the Supplemental Material [22]. It would be also interesting to perform numerical calculation on our network model with interactions, to confirm our predictions.

We thank Peter Abbamonte, Ehud Altman, Liang Fu, Hiroshi Fukuyama, Jung-Hoon Han, Tim Hsieh, Eun-Ah Kim, Yong Baek Kim, Jong Hwan Kim, Hae Young Kee, Tae Hwan Kim, Sungbin Lee, Ivar Martin, Masashi Morishita, Adrian Po, Youngwoo Son, Dam T. Son, Ashvin Vishwanath, and Mike Zaletel for helpful discussion. The research of S.L. was supported by NSERC. Research at Perimeter Institute is supported in part by the Government of Canada through the Department of Innovation, Science and Economic Development Canada and by the Province of Ontario through the Ministry of Colleges and Universities. C. G. and M. O. were supported in part by MEXT/JSPS KAKENHI Grants No. JP18H03686 and No. JP17H06462. J. W. P. and H. W. Y are supported by the Institute for Basic Science (IBSR014-D1). G. Y. C thanks for the support of the Visiting Fellowship at the Perimeter Institute. Part of this work is done during the winter program "New Approaches to Strongly Correlated Quantum Systems" at Aspen Center for Physics, supported by the U.S. National Science Foundation Grant No. NSF PHY-1607611, and at the Kavli Institute for Theoretical Physics, UC Santa Barbara, supported by NSF PHY-1748958. This work was supported by the National Research Foundation of
Korea (NRF) grant funded by the Korea government (MSIT) (No. 2020R1C1C1006048).

Note added.- Recently, we became aware of independent work on decorated star lattices [68] where flatbands and higher-order topology are also discussed. We thank Hosho Katsura for several useful comments and bringing our attention to this recent independent paper [68] and informing us about Ref. [35], where the energy of the flatbands is shown to be related to the energy of a single wire subject under the open boundary condition.

*gilyoungcho@postech.ac.kr

[1] M.Z. Hasan and C. L. Kane, Colloquium: Topological insulators, Rev. Mod. Phys. 82, 3045 (2010).

[2] X.-L. Qi and S.-C. Zhang, Topological insulators and superconductors, Rev. Mod. Phys. 83, 1057 (2011).

[3] N. Shima and H. Aoki, Electronic Structure of SuperHoneycomb Systems: A Peculiar Realization of Semimetal/Semiconductor Classes and Ferromagnetism, Phys. Rev. Lett. 71, 4389 (1993).

[4] A. Ramachandran, A. Andreanov, and S. Flach, Chiral flat bands: Existence, engineering, and stability, Phys. Rev. B 96, 161104(R) (2017).

[5] Y. Cao, V. Fatemi, S. Fang, K. Watanabe, T. Taniguchi, E. Kaxiras, and P. Jarillo-Herrero, Unconventional superconductivity in magic-angle graphene superlattices, Nature (London) 556, 43 (2018).

[6] Y. Cao, V. Fatemi, A. Demir, S. Fang, S. L. Tomarken, J. Y. Luo, J. D. Sanchez-Yamagishi, K. Watanabe, T. Taniguchi, E. Kaxiras et al., Correlated insulator behaviour at half-filling in magic-angle graphene superlattices, Nature (London) 556, 80 (2018).

[7] R. Bistritzer and A. H. MacDonald, Moiré bands in twisted double-layer graphene, Proc. Natl. Acad. Sci. U.S.A. 108, 12233 (2011).

[8] J. W. Park, G. Y. Cho, J. Lee, and H. W. Yeom, Emergent honeycomb network of topological excitations in correlated charge density wave, Nat. Commun. 10, 1 (2019).

[9] J. A. Wilson, F. J. Di Salvo, and S. Mahajan, Charge-density waves and superlattices in the metallic layered transition metal dichalcogenides, Adv. Phys. 24, 117 (1975).

[10] B. Sipos, A. F. Kusmartseva, A. Akrap, H. Berger, L. Forró, and E. Tutiš, From Mott state to superconductivity in $1 \mathrm{~T}-\mathrm{TaS}_{2}$, Nat. Mater. 7, 960 (2008).

[11] Y. Yu, F. Yang, X. F. Lu, Y. J. Yan, Y.-H. Cho, L. Ma, X. Niu, S. Kim, Y.-W. Son, D. Feng et al., Gate-tunable phase transitions in thin flakes of $1 \mathrm{~T}-\mathrm{TaS}_{2}$, Nat. Nanotechnol. 10, 270 (2015).

[12] Y. Liu, D. F. Shao, L. J. Li, W. J. Lu, X. D. Zhu, P. Tong, R. C. Xiao, L. S. Ling, C. Y. Xi, L. Pi, H. F. Tian, H. X. Yang, J. Q. Li, W. H. Song, X. B. Zhu, and Y. P. Sun, Nature of charge density waves and superconductivity in $1 T-\mathrm{TaSe}_{2-x} \mathrm{Te}_{x}$, Phys. Rev. B 94, 045131 (2016).

[13] P. Fazekas and E. Tosatti, Charge carrier localization in pure and doped 1T-TaS 2 , Physica (Amsterdam) 99(B+C), 183 (1980). 
[14] K. T. Law and P. A. Lee, $1 \mathrm{~T}-\mathrm{TaS}_{2}$ as a quantum spin liquid, Proc. Natl. Acad. Sci. U.S.A. 114, 6996 (2017).

[15] W.-Yu. He, X. Y. Xu, G. Chen, K. T. Law, and P. A. Lee, Spinon Fermi Surface in a Cluster Mott Insulator Model on a Triangular Lattice and Possible Application to $1 \mathrm{~T}-\mathrm{TaS}_{2}$, Phys. Rev. Lett. 121, 046401 (2018).

[16] L. J. Li, E. C. T. Ofarrell, K. P. Loh, G. Eda, B. Özyilmaz, and A.H.C. Neto, Controlling many-body states by the electric-field effect in a two-dimensional material, Nature (London) 529, 185 (2016).

[17] C. Chen, L. Su, A. H. C. Neto, and V. M. Pereira, Discommensuration-driven superconductivity in the charge density wave phases of transition-metal dichalcogenides, Phys. Rev. B 99, 121108(R) (2019).

[18] A. Spijkerman, J. L. de Boer, A. Meetsma, G. A. Wiegers, and S. van Smaalen, X-ray crystal-structure refinement of the nearly commensurate phase of $1 \mathrm{~T}-\mathrm{TaS}_{2}$ in $(3+2)$ dimensional superspace, Phys. Rev. B 56, 13757 (1997).

[19] X. L. Wu and C. M. Lieber, Hexagonal domain-like charge density wave phase of $\mathrm{TaS}_{2}$ determined by scanning tunneling microscopy, Science 243, 1703 (1989).

[20] M. Morishita, Fluidity in domain walls in dilute ${ }^{3} \mathrm{He}-{ }^{4} \mathrm{He}$ films on graphite: Possible 1D fermi fluid and Dirac fermions in helium film, arXiv:1908.01991.

[21] B. Dóra, J. Kailasvuori, and R. Moessner, Lattice generalization of the dirac equation to general spin and the role of the flat band, Phys. Rev. B 84, 195422 (2011).

[22] See Supplemental Material at http://link.aps.org/ supplemental/10.1103/PhysRevLett.124.137002 for details. The references of Supplemental Material include Refs. [8,23-32].

[23] J. Skolimowski, Y. Gerasimenko, and R. Žitko, Mottness Collapse Without Metallization in the Domain Wall of the Triangular-Lattice Mott Insulator $1 \mathrm{~T}-\mathrm{TaS}_{2}$, Phys. Rev. Lett. 122, 036802 (2019).

[24] W. A. Benalcazar, B. A. Bernevig, and T. L. Hughes, Quantized electric multipole insulators, Science 357, 61 (2017).

[25] F. Schindler, A. M. Cook, M. G. Vergniory, Z. Wang, S. S. P. Parkin, B. A. Bernevig, and T. Neupert, Higher-order topological insulators, Sci. Adv. 4, eaat0346 (2018).

[26] F. Schindler, Z. Wang, M. G. Vergniory, A. M. Cook, A. Murani, S. Sengupta, A. Yu. Kasumov, R. Deblock, S. Jeon, I. Drozdov, H. Bouchiat, S. Guron, A. Yazdani, B. A. Bernevig, and T. Neupert, Higher-order topology in bismuth, Nat. Phys. 14, 918 (2018).

[27] D. Cho, G. Gye, J. Lee, S.-H. Lee, L. Wang, S.-W. Cheong, and H.W. Yeom, Correlated electronic states at domain walls of a Mott-charge-density-wave insulator $1 \mathrm{~T}-\mathrm{TaS}_{2}$, Nat. Commun. 8, 392 (2017).

[28] D. K. Efimkin and A. H. MacDonald, Helical network model for twisted bilayer graphene, Phys. Rev. B 98, 035404 (2018).

[29] J. T. Chalker and P. D. Coddington, Percolation, quantum tunnelling and the integer hall effect, J. Phys. C 21, 2665 (1988).

[30] K. Sun, H. Yao, E. Fradkin, and S. A. Kivelson, Topological Insulators and Nematic Phases from Spontaneous Symmetry Breaking in 2d Fermi Systems with a Quadratic Band Crossing, Phys. Rev. Lett. 103, 046811 (2009).
[31] B. Kang, K. Shiozaki, and G. Y. Cho, Many-body invariants for multipoles in higher-order topological insulators, Phys. Rev. B 100, 245134 (2019).

[32] Y.-Q. Wang and J.E. Moore, Boundary edge networks induced by bulk topology, Phys. Rev. B 99, 155102 (2019).

[33] D. Leykam, A. Andreanov, and S. Flach, Artificial flat band systems: From lattice models to experiments, Adv. Phys. X 3, 1473052 (2018).

[34] D. L. Bergman, C. Wu, and L. Balents, Band touching from real-space topology in frustrated hopping models, Phys. Rev. B 78, 125104 (2008).

[35] H. Katsura and I. Maruyama (private communication).

[36] E. Tang, J.-W. Mei, and X.-G. Wen, High-Temperature Fractional Quantum Hall States, Phys. Rev. Lett. 106, 236802 (2011).

[37] T. Ritschel, J. Trinckauf, K. Koepernik, B. Büchner, M. v. Zimmermann, H. Berger, Y. I. Joe, P. Abbamonte, and J. Geck, Orbital textures and charge density waves in transition metal dichalcogenides, Nat. Phys. 11, 328 (2015).

[38] T. Ritschel, H. Berger, and J. Geck, Stacking-driven gap formation in layered $1 \mathrm{~T}-\mathrm{TaS}_{2}$, Phys. Rev. B 98, 195134 (2018).

[39] L. Le Guyader, T. Chase, A. H. Reid, R. K. Li, D. Svetin, X. Shen, T. Vecchione, X. J. Wang, D. Mihailovic, and H. A. Dürr, Stacking order dynamics in the quasi-twodimensional dichalcogenide $1 \mathrm{~T}-\mathrm{TaS}_{2}$ probed with $\mathrm{MeV}$ ultrafast electron diffraction, Struct. Dyn. 4, 044020 (2017).

[40] S.-H. Lee, J. S. Goh, and D. Cho, Origin of the Insulating Phase and First-Order Metal-Insulator Transition in 1T-TaS 2 , Phys. Rev. Lett. 122, 106404 (2019).

[41] P. D. Hambourger and F. J. Di Salvo, Electronic conduction process in $1 \mathrm{~T}-\mathrm{TaS}_{2}$, Physica (Amsterdam) 99(B+C), 173 (1980).

[42] D. Svetin, I. Vaskivskyi, S. Brazovskii, and D. Mihailovic, Three-dimensional resistivity and switching between correlated electronic states in $1 \mathrm{~T}-\mathrm{TaS}_{2}$, Sci. Rep. 7, 46048 (2017).

[43] D. Kühn, M. Müller, F. Sorgenfrei, E. Giangrisostomi, R. M. Jay, R. Ovsyannikov, N. Mårtensson, D. Sánchez-Portal, and A. Föhlisch, Directional sub-femtosecond charge transfer dynamics and the dimensionality of $1 \mathrm{~T}-\mathrm{TaS}_{2}$, Sci. Rep. 9, 488 (2019).

[44] A. Julku, S. Peotta, T. I. Vanhala, D.-H. Kim, and P. Törmä, Geometric Origin of Superfluidity in the Lieb-Lattice Flat Band, Phys. Rev. Lett. 117, 045303 (2016).

[45] H. Tasaki, Ferromagnetism in the Hubbard Models with Degenerate Single-Electron Ground States, Phys. Rev. Lett. 69, 1608 (1992).

[46] R. Mondaini, G. G. Batrouni, and B. Grémaud, Pairing and superconductivity in the flat band: Creutz lattice, Phys. Rev. B 98, 155142 (2018).

[47] E. W. Huang, M.-S. Vaezi, Z. Nussinov, and A. Vaezi, Enhanced correlations and superconductivity in weakly interacting partially flat-band systems: A determinantal quantum monte carlo study, Phys. Rev. B 99, 235128 (2019).

[48] H. Tasaki, Stability of Ferromagnetism in the Hubbard Model, Phys. Rev. Lett. 73, 1158 (1994). 
[49] A. Y. Liu, Electron-phonon coupling in compressed $1 \mathrm{~T}-\mathrm{TaS}_{2}$ : Stability and superconductivity from first principles, Phys. Rev. B 79, 220515 (2009).

[50] P. Lazar, J. Martincová, and M. Otyepka, Structure, dynamical stability, and electronic properties of phases in $\mathrm{TaS}_{2}$ from a high-level quantum mechanical calculation, Phys. Rev. B 92, 224104 (2015).

[51] X.-C. Wu, C.-M. Jian, and C. Xu, Coupled-wire description of the correlated physics in twisted bilayer graphene, Phys. Rev. B 99, 161405(R) (2019).

[52] E. Fradkin, Field Theories of Condensed Matter Physics (Cambridge University Press, Cambridge, England, 2013).

[53] S. A. Kivelson, E. Fradkin, and V. J. Emery, Electronic liquid-crystal phases of a doped Mott insulator, Nature (London) 393, 550 (1998).

[54] A. Tokuno, M. Oshikawa, and E. Demler, Dynamics of OneDimensional Bose Liquids: Andreev-Like Reflection at $y$ Junctions and the Absence of the Aharonov-Bohm Effect, Phys. Rev. Lett. 100, 140402 (2008).

[55] C. Chamon, M. Oshikawa, and I. Affleck, Junctions of Three Quantum Wires and the Dissipative Hofstadter Model, Phys. Rev. Lett. 91, 206403 (2003).

[56] M. Oshikawa, C. Chamon, and I. Affleck, Junctions of three quantum wires, J. Stat. Mech. (2006) P02008.

[57] J. N. Reimers and A. J. Berlinsky, Order by disorder in the classical heisenberg kagomé antiferromagnet, Phys. Rev. B 48, 9539 (1993).

[58] E. Berg, E. Fradkin, S. A. Kivelson, and J. M. Tranquada, Striped superconductors: How spin, charge and superconducting orders intertwine in the cuprates, New J. Phys. 11, 115004 (2009).

[59] Y. Ma, S. Kolekar, H. C. Diaz, J. Aprojanz, I. Miccoli, C. Tegenkamp, and M. Batzill, Metallic twin grain boundaries embedded in $\mathrm{MoSe}_{2}$ monolayers grown by molecular beam epitaxy, ACS Nano 11, 5130 (2017).
[60] C. Barreteau, F. Ducastelle, and T. Mallah, A birds eye view on the flat and conic band world of the honeycomb and kagome lattices: Towards an understanding of $2 \mathrm{~d}$ metalorganic frameworks electronic structure, J. Phys. Condens. Matter 29, 465302 (2017).

[61] H. Sun, B. Li, and J. Zhao, Half-metallicity in 2d organometallic honeycomb frameworks, J. Phys. Condens. Matter 28, 425301 (2016).

[62] L. Zheng, L. Feng, and W. Yong-Shi, Exotic electronic states in the world of flat bands: From theory to material, Chin. Phys. B 23, 077308 (2014).

[63] M. Maruyama, N. Thanh Cuong, and S. Okada, Coexistence of dirac cones and kagome flat bands in a porous graphene, Carbon 109, 755 (2016).

[64] Q. Hu, C. Yin, L. Zhang, L. Lei, Z. Wang, Z. Chen, J. Tang, and R. Ang, Direct observation of melted Mott state evidenced from raman scattering in $1 \mathrm{~T}-\mathrm{TaS}_{2}$ single crystal, Chin. Phys. B 27, 017104 (2018).

[65] L. Perfetti, P. A. Loukakos, M. Lisowski, U. Bovensiepen, H. Berger, S. Biermann, P. S. Cornaglia, A. Georges, and M. Wolf, Time Evolution of the Electronic Structure of $1 \mathrm{~T}-\mathrm{TaS}_{2}$ Through the Insulator-Metal Transition, Phys. Rev. Lett. 97, 067402 (2006).

[66] C. Sohrt, A. Stange, M. Bauer, and K. Rossnagel, How fast can a Peierls-Mott insulator be melted?, Faraday Discuss. 171, 243 (2014).

[67] P. Rickhaus, J. Wallbank, S. Slizovskiy, R. Pisoni, H. Overweg, Y. Lee, M. Eich, M.-H. Liu, K. Watanabe, T. Taniguchi et al., Transport through a network of topological channels in twisted bilayer graphene, Nano Lett. 18, 6725 (2018).

[68] T. Mizoguchi, M. Maruyama, S. Okada, and Y. Hatsugai, Flat bands and higher-order topology in polymerized triptycene: Tight-binding analysis on decorated star lattices, Phys. Rev. Mater. 3, 114201 (2019). 